\title{
Estimates of HE-LHC beam parameters at different injection energies
}

\author{
Tanaji Sen \\ Accelerator Physics Center \\ FNAL, Batavia, IL 60510, USA
}

\begin{abstract}
A future upgrade to the LHC envisions increasing the top energy to $16.5 \mathrm{TeV}$ and upgrading the injectors. There are two proposals to replace the SPS as the injector to the LHC. One calls for a superconducting ring in the SPS tunnel while the other calls for an injector (LER) in the LHC tunnel. In both scenarios, the injection energy to the LHC will increase. In this note we look at some of the consequences of increased injection energy to the beam dynamics in the LHC.
\end{abstract}

\section{INTRODUCTION}

The present design luminosity of the LHC is $10^{34} \mathrm{~cm}^{-2} \mathrm{~s}^{-1}$ at a top energy of $7 \mathrm{TeV}$. Upgrades to the LHC are envisaged to proceed in two steps - first the luminosity will be increased (the so-called HL-LHC) and next the top energy more than doubled to 16.5 $\mathrm{TeV}$ (the so-called HE-LHC). This ambitious program will also require upgrades to many of the injectors, especially the SPS. One plan is to build a fast cycling superconducting accelerator (the S-SPS) in the same tunnel as the SPS. This new injector would accept beams from the SPS and accelerate them to 1 or $1.3 \mathrm{TeV}$ before extraction to the LHC. Another recently proposed option [1] is to build an injector (the LER) in the LHC ring with transmission line style magnets, similar to the ones proposed for the VLHC. The LER will be capable of accelerating beams to $1.65 \mathrm{TeV}$. There are many significant differences between the two options both in construction and beam issues in the two accelerators. One of the major beam issues is the maximum beam energy that can be injected into the LHC. In this note we will consider the impact of a change in the beam injection energy on beam dynamics in the LHC. No detailed simulations will be done but simple formulas will be used to extract the energy dependence of the relevant quantities. Table 1 shows the values of some basic LHC beam parameters.

Table 1: Input parameters of the LHC

\begin{tabular}{|c|c|}
\hline Parameter & Value \\
\hline Nominal Bunch intensity & $1.15 \times 10^{11}$ \\
Number of bunches & 2808 \\
Transverse emittance $(1 \sigma)[\mathrm{mm}-\mathrm{mrad}]$ & 3.75 \\
Longitudinal emittance $(4 \sigma)[\mathrm{eV}-\mathrm{sec}]$ & 1.0 \\
Rf frequency $[\mathrm{MHz}]$ & 400 \\
RF voltage at injection $[\mathrm{MV}]$ & 8.0 \\
& \\
\hline
\end{tabular}


Higher injection energy will affect several key beam dynamics issues. These include (1) Dynamic Aperture, (2) Persistent current decay and snapback, (3) Instabilities, (4) Electron cloud, (5) Intra-beam scattering, (6) Synchrotron radiation, (7) Rest-gas scattering and possibly others.

\section{DYNAMIC APERTURE}

At injection energy, the field quality of the superconducting dipoles in the main arcs poses the strongest limits to the dynamic aperture. Increasing the beam energy will help to increase the dynamic aperture for two reasons

- the beam size decreases with increasing energy as $1 / \sqrt{\gamma}$, hence the physical aperture will be larger when measured in units of the rms beam size

- the field quality improves with increasing energy. This will also result in a larger dynamic aperture.

As an example, increasing the beam energy from $0.45 \mathrm{TeV}$ to $1.65 \mathrm{TeV}$ in the LHC will reduce the beam size by 1.9 times. Quantifying the increase due to the second effect will require field quality measurements at different energies and particle tracking. For a cruder estimate, scaling laws could be applied to estimate the impact on the dynamic aperture if the multipole errors in the magnets at higher energies are known.

\section{PERSISTENT CURRENT DECAY AND SNAPBACK}

At the injection plateau, persistent currents in the superconducting magnets decay with time. This decay in the main field is also accompanied by decays in the multipole components, specifically the sextupole $\left(b_{3}\right)$ and decapole $\left(b_{5}\right)$ components in the main dipoles. The time constant for this decay depends on several factors including the initial magnetic field, the cable, the magnet's history etc. A quantitative model for this decay does not seem to exist [2]. The persistent currents in the LHC magnets decay by about a third [3] during the injection plateau which lasts several minutes. At the start of the ramp, the fields snap back to their initial values on a much shorter time scale, of the order of seconds in the LHC. It is estimated that $b_{3}$ changes by more than 3 units during this process leading to a chromaticity change of more than 150 units. In addition to the chromaticity change which is the dominant effect on the beam, there are also smaller relative changes in the orbit, tunes, beta beats and collimation efficiency. Correction algorithms have been devised to ensure that these changes have little impact on the beam.

At higher injection energy and hence higher fields, the persistent currents decay at a slower rate. Consequently the amount of snapback will be smaller and the impact on the beam will also be reduced.

Measurements of the sextupole current in a dipole magnet with initial field 1.2T corresponding to an energy of $1 \mathrm{TeV}$ showed that the persistent current decay was reduced by a factor of 2.6 [5]. One expects that at a higher field of $2 \mathrm{~T}$ corresponding to 
1.65 TeV, the decay would be even less. This would help reduce the setup time, overall turn around time between luminosity stores and help increase the integrated luminosity.

\section{INSTABILITIES}

Here we will estimate the impact of raising the injection energy on the important instabilities at injection. The formulae in this section are taken from reference [4].

\section{IV.A Direct space charge tune shift and tune spread}

The direct space charge creates an incoherent tune spread with smaller tune shifts for larger amplitude particles. The tune shift at small amplitudes is

$$
\Delta Q_{d s c}=\frac{N_{b} r_{p}}{4 \pi B \varepsilon_{N}} \frac{1}{\gamma^{2}}
$$

Notation: $N_{b}$ = bunch intensity, $r_{p}=$ classical proton radius, $B=$ bunching factor, $\varepsilon_{N}=$ normalized transverse emittance, $\gamma$ is the relativistic factor.

This direct space charge effect decreases rapidly with the inverse square of the energy. At $450 \mathrm{GeV}$, this tune shift and tune spread is $\sim 0.001$ and comparable to the tune spread from the lattice nonlinearities. This tune spread may also help contribute to the Landau damping of mode numbers higher than the rigid dipole mode [6]. The fact that the space charge tune spread will be an order of magnitude smaller at $1.65 \mathrm{TeV}$ should not be an issue since there is a transverse feedback system in the LHC.

\section{IV.B Laslett tune shift}

Due to the image current induced on the beam pipe and ferromagnetic magnet poles; all particles suffer a tune shift with opposite sign in the two transverse planes. In the vertical plane, the shift is

$$
\Delta Q_{y, \text { Laslett }}=-\frac{N_{b} k_{b} r_{p} \beta_{a v}}{\pi}\left(\frac{\varepsilon_{1}}{h^{2}}+\frac{\varepsilon_{2}}{g^{2}}\right) \frac{1}{\gamma}
$$

Notation: $k_{b}=$ Number of bunches, $\beta_{a v}=$ average beta function around the ring, $\varepsilon_{1}, \varepsilon_{2}=$ Electric and magnetic Laslett coefficients which depend on the geometry, $h=$ half-height of beam pipe, $\mathrm{g}=$ half the distance between magnet poles

At $450 \mathrm{GeV}$, this shift is about 0.01 which is significant. This tune shift is likely compensated by the tuning quadrupoles. The Laslett tune shift decreases inversely with the energy and will require smaller changes in the tuning quadrupoles at higher energy.

\section{IV.C Space charge impedance}

Space charge also creates a capacitive coupling impedance that depends on the sizes of the beam pipe and the beam. Assuming a circular beam pipe of radius $b$ and beam radius $a=\sqrt{\beta_{a v} \varepsilon}$, the longitudinal and transverse coupling impedances are 


$$
\begin{aligned}
& Z_{L}(\omega)=-j \frac{\omega R Z_{0}}{2 c}\left[1+2 \ln \left(\frac{b}{a}\right)\right] \frac{1}{\gamma^{2}} \quad \propto \frac{1}{\gamma^{2}} \\
& Z_{T}(\omega)=-j Z_{0} R\left(\frac{1}{2 a^{2}}-\frac{1}{b^{2}}\right) \frac{1}{\gamma^{2}} \quad \propto \frac{1}{\gamma} \quad \text { assuming } a<b b
\end{aligned}
$$

Notation: $R$ is the average machine radius, $Z_{0}=376.73 \mathrm{Ohms}$ is the impedance of free space. These give rise to coherent tune shifts in the longitudinal and transverse planes which can be estimated using these impedances as effective impedances.

\section{IV.D Coherent tune shifts due to space charge}

The longitudinal complex tune shift in the presence of complex impedances is

$$
\Delta Q_{L}^{(m, n)}=-j \frac{|n|}{|n|+1} \frac{Q_{s} I_{b}}{3 h_{r f} V_{r f}}\left(\frac{2 \pi R}{L_{s}}\right)^{3}\left(\frac{Z_{L}}{n}\right)_{e f f}^{(m, n)} \propto\left(\frac{Z_{L}}{n}\right)_{e f f}^{(m, n)} \gamma^{1 / 4}
$$

Notation: $n= \pm 1, \pm 2$, is the azimuthal mode number and $m=0,1, k_{b}-1$ is the coupled bunch mode number. $Q_{s}$ is the synchrotron tune, $I_{b}$ is the bunch current, and $L_{s}=4 \sigma_{s}$ is the full bunch length.

In extracting the energy dependence, we used the relations $Q_{s} \propto \gamma^{-1 / 2}$ while $L_{s} \propto \gamma^{-1 / 4}$. The dependence of the effective impedance on the energy is determined mainly by the frequency dependent impedance but it is also modified by the bunch spectrum.

The transverse complex tune shift is

$$
\Delta Q_{T}^{(m, n)}=\frac{j}{|n|+1} \frac{I_{b} R}{2(E / e) L_{s}} \beta_{a v}\left(Z_{T}\right)_{e f f}^{(m, n)} \propto\left(Z_{T}\right)_{e f f}^{(m, n)} \frac{1}{\gamma^{3 / 4}}
$$

Here $(E / e)$ is the beam energy in volts.

The effective impedances are found by averaging the frequency dependent impedances over the bunch mode spectrum. The relative transverse tune shifts $\Delta Q_{T}^{(m, n)} /\left\{Q_{\beta}\right\}$, where $\left\{Q_{\beta}\right\}$ is the fractional part of the betatron tune, for some low order modes at $450 \mathrm{GeV}$ are of the order of $10^{-3}$ and the relative longitudinal tune shifts for low order modes are of the order of $10^{-4}$. At higher energies, these shifts become even smaller and are negligible.

\section{IV.E Longitudinal microwave instability threshold intensity}

The threshold for the longitudinal microwave instability is

$$
I_{b}^{\text {th }} \approx \frac{3}{2} \frac{h_{r f} V_{r f}}{\left(Z_{L} / n\right)_{\text {eff }}}\left(\frac{L_{s}}{2 \pi R}\right)^{3} \propto \frac{1}{\gamma^{3 / 4}} \frac{1}{\left(Z_{L} / n\right)_{\text {eff }}}
$$


The threshold intensity decreases with increasing energy so this would be an argument against higher injection energy if this threshold is close to realistic bunch intensities. As it turns out, the threshold is far above ultimate intensities in the LHC so this does not pose a concern.

\section{IV.F Loss of Landau damping against longitudinal instabilities}

The longitudinal coherent tune shifts should not exceed the longitudinal tune spread for longitudinal Landau damping to be maintained. Requiring that the tune shift be less than (tune spread)/4 imposes the intensity threshold

$$
I_{b}^{t h} \approx \frac{3 \pi^{2}}{32} \frac{h_{r f}^{3} V_{r f}}{\operatorname{Im}\left[\left(Z_{L} / n\right)_{e f f}^{b b}\right]}\left(\frac{L_{s}}{2 \pi R}\right)^{5} \propto \frac{1}{\gamma^{5 / 4}} \frac{1}{\operatorname{Im}\left[\left(Z_{L} / n\right)_{e f f}^{b b}\right]}
$$

This threshold also decreases with increasing energy and faster than the threshold for the longitudinal microwave instability. The longitudinal effective broadband impedance has a negligibly small dependence on energy. Among all the instabilities considered here, this instability imposes the smallest threshold intensity at $7 \mathrm{TeV}$ of about $9.1 \times 10^{11}$ particles/bunch, assuming an effective broadband impedance of $0.076 \mathrm{~m} \Omega$. This threshold is still sufficiently above feasible intensities that a dedicated longitudinal feedback system was not considered necessary [7].

\section{IV.G Transverse Mode Coupling Instability}

This occurs when two neighboring head-tail modes coalesce. The threshold is given by

$$
I_{b}^{\text {th }} \approx \frac{2 Q_{s} E}{e \beta_{a v} \operatorname{Im}\left[Z_{T}\right]_{e f f}} \frac{L}{R} \propto \frac{\gamma^{1 / 4}}{\operatorname{Im}\left[Z_{T}\right]_{e f f}}
$$

Unlike the other instabilities, the threshold for this instability increases with energy. This is especially beneficial since the $\mathrm{TMCl}$ instability has the lowest threshold among the three instabilities at $450 \mathrm{GeV}$.

The CERN design report [8] states that the imaginary part of the effective broad-band impedance is $1.34 \mathrm{M} \Omega / \mathrm{m}$ at $450 \mathrm{GeV}$ and rises to $2.67 \mathrm{M} \Omega / \mathrm{m}$ at $7 \mathrm{TeV}$. This is a fairly slow increase, hence in the absence of a detailed knowledge of the impedance values, we will use the value of $1.5 \mathrm{M} \Omega / \mathrm{m}$ at all injection energies.

\section{IV.G Coupled bunch resistive wall instability}

Coupled bunch instabilities can be driven by the narrow band transverse resistive wall impedances. Given the impedance $Z_{T}$ at the frequency of the lowest unstable mode, the growth time of the instability can be found from 
$\frac{1}{\tau_{R W}}=\frac{N_{b} k_{b} r_{0} \omega_{0}^{2}}{2 \pi \gamma c Z_{0}} \beta_{a v} \operatorname{Re}\left[Z_{T}(\omega)\right] \quad \propto \quad \frac{\operatorname{Re}\left[Z_{T}(\omega)\right]}{\gamma}$

In the LHC design report [8], the magnitude of the real part of the transverse impedance at the lowest unstable mode, $8 \mathrm{kHz}$, increases from $57 \mathrm{MOhm} / \mathrm{m}$ at $450 \mathrm{GeV}$ to $145 \mathrm{MOhm} / \mathrm{m}$ at $7 \mathrm{GeV}$, almost a factor of 3 over an energy that increases by nearly a factor of 16. Without a detailed impedance model, it is not possible to calculate the impedance at the higher energies but the increase will be slower than a linear growth. The growth time of the vertical instability will therefore increase from the present estimate of $27 \mathrm{msec}$ at $450 \mathrm{GeV}$ [8] as the energy is increased to $1.65 \mathrm{TeV}$ but by less than a factor of 4 . The requirements of the transverse feedback system will therefore be relaxed at the higher energies.

\section{IV.I Summary of instabilities}

Table 2: Tune shifts, impedances and threshold at different energies

\begin{tabular}{|c|c|c|c|c|}
\hline & $\begin{array}{l}\text { Energy } \\
\text { scaling }\end{array}$ & $450 \mathrm{GeV}$ & $1 \mathrm{TeV}$ & 1.65TeV \\
\hline RMS bunch length $[\mathrm{cm}]$ & $E^{-1 / 4}$ & 11.24 & 9.23 & 8.15 \\
\hline RMS energy spread & $E^{-3 / 4}$ & $4.72 \times 10^{-4}$ & $2.58 \times 10^{-4}$ & $1.77 \times 10^{-4}$ \\
\hline Direct space charge tune shift & $E^{-2}$ & $-1.54 \times 10^{-3}$ & $-3.8 \times 10^{-4}$ & $-1.58 \times 10^{-4}$ \\
\hline Laslett tune shift & $E^{-1}$ & $-1.42 \times 10^{-2}$ & $-6.4 \times 10^{-3}$ & $-3.88 \times 10^{-3}$ \\
\hline $\begin{array}{l}\text { Space charge transverse } \\
\text { impedance }[\mathrm{M} \Omega / \mathrm{m}]\end{array}$ & $E^{-1}$ & -j 6.71 & $-j 3.03$ & -j 1.83 \\
\hline $\begin{array}{l}\text { Space charge long. Impedance } \\
{[\mathrm{m} \Omega]}\end{array}$ & $E^{-2}$ & $-j 6.04$ & - i 1.36 & -0.528 \\
\hline Microwave threshold intensity & $\mathrm{E}^{-3 / 4}$ & $1.14 \times 10^{13}$ & $6.3 \times 10^{12}$ & $4.3 \times 10^{12}$ \\
\hline $\begin{array}{l}\text { Landau damping threshold } \\
\text { intensity (longitudinal) }\end{array}$ & $E^{-5 / 4}$ & $2.5 \times 10^{12}$ & $9.5 \times 10^{11}$ & $5.1 \times 10^{11}$ \\
\hline $\mathrm{TMCl}$ threshold intensity & $E^{1 / 4}$ & $3.0 \times 10^{12}$ & $3.7 \times 10^{12}$ & $4.2 \times 10^{12}$ \\
\hline
\end{tabular}

\section{Comments:}

- The rms energy spread decreases rapidly with increasing energy - at $1.65 \mathrm{TeV}$ the spread is less than half the value at $450 \mathrm{GeV}$. This should make injection easier, reduce the sensitivity to chromatic errors, help collimation efficiency, improve the lifetime and may also improve the ramp efficiency.

- The space charge tune shifts (direct and Laslett) as well the space charge impedances fall with increasing energy. Space charge related issues may not be a factor in the range of higher injection energies considered.

- The space charge impedances are capacitive, indicated by the (-j)

- Threshold intensities are quoted for the bunch intensities. The variation of the threshold intensities with energy does not include the slower dependence of the 
effective impedances on the energy. It also assumes parameters such as the rf voltage are held constant.

- The microwave instability threshold intensity and the longitudinal Landau damping threshold intensity are found by assuming $\left(Z_{L} / n\right)$ _eff $=0.1 \Omega$, The $T M C l$ threshold intensity is found assuming a transverse impedance $Z_{T}=1.5 \mathrm{M} \Omega / \mathrm{m}$.

For more accurate values of the LHC impedances, appropriate corrections should be made.

- The thresholds for the longitudinal microwave and the loss of Landau damping against longitudinal instabilities fall with increasing energy. The threshold for the loss of Landau damping is the more critical. However even at $1.65 \mathrm{TeV}$, the threshold intensity at $5.1 \times 10^{11}$ is well above ultimate intensity. Note also that the realistic value of the effective longitudinal broad-band impedance may be smaller than 0.1 Ohms as assumed.

- The TMCl threshold by contrast increases with energy.

\section{ELECTRON CLOUD}

At injection energies in the range $450 \mathrm{GeV}-1.65 \mathrm{TeV}$, the synchrotron radiation photons are not energetic enough to produce photoelectrons when they strike the beam chamber. Instead, electrons are produced either by gas ionization or lost protons. The electron cloud density should be smaller than at $7 \mathrm{TeV}$. However the wakefields from the electron cloud may have a stronger effect at lower energies.

Threshold e-cloud density for the single bunch instability is given by [8]

$$
\rho_{e, t h r} \approx \frac{2 \gamma Q_{s}}{\pi \beta r_{p} C} \propto \gamma^{1 / 2}
$$

Increasing the injection energy will also beneficially increase the threshold electron density for the instability to develop.

Rise time of instability from the coupled bunch instability is [8]

$$
\tau_{e, C B} \approx \frac{\gamma}{2 \pi r_{p} c \beta \rho_{e}} \propto \frac{\gamma}{\rho_{e}}
$$

where $\rho_{e}$ is the electron cloud density assumed to be above threshold for the instability. This rise time increases with energy, thus a higher injection energy may slow down the growth of the coupled bunch instability due to the electron cloud.

A recent study [9], reporting numerical simulations and an experimental measurement in the SPS, suggests that the threshold for the electron cloud instability may not have the simple behaviour predicted by the above expressions. Instead the study claims that the smaller transverse beam size at higher energies enhances the electron cloud pinch and the smaller synchrotron tune implies longer damping times. The study suggests that the intensity threshold reaches a constant value above a certain energy. It is not yet clear if these results are true in general and in particular if they apply to the LHC at the injection energies of interest. 


\section{SYNCHROTRON RADIATION}

Table 3 shows some of the relevant parameters related to synchrotron radiation from the beam at different injection energies.

Table 3: Some synchrotron radiation parameters

\begin{tabular}{|l|l|l|l|}
\hline & $450 \mathrm{GeV}$ & $1 \mathrm{TeV}$ & $1.65 \mathrm{TeV}$ \\
\hline $\begin{array}{l}\text { Synchrotron radiation power from beam per } \\
\text { ring [W] }\end{array}$ & 0.066 & 1.61 & 11.9 \\
Energy loss per particle per turn [eV] & 0.114 & 2.78 & 20.6 \\
Critical photon energy [eV] & 0.0117 & 0.128 & 0.574 \\
Average photon energy [eV] & 0.0036 & 0.039 & 0.177 \\
Transverse damping time [hrs] & 97537 & 8896 & 1980 \\
\hline
\end{tabular}

Effects of synchrotron radiation should not have a significant effect on the beam at these injection energies. The critical energy even at $1.65 \mathrm{TeV}$ is well below the work function of photo-electrons ( $40 \mathrm{eV}$ ) so there should be no significant increase in photoelectrons at $1.65 \mathrm{TeV}$.

\section{INTRA-BEAM SCATTERING}

Intra-beam scattering contributes to emittance growth, the growth of the longitudinal emittance at high energy is mainly due to intra-beam scattering. To estimate the energy dependence, we will use the simplified expressions from reference [10]. The growth rate of the energy spread is given by

$$
\begin{aligned}
& \frac{1}{T_{p}} \equiv \frac{1}{\sigma_{p}} \frac{d \sigma_{p}}{d t} \approx \frac{r_{0}^{2} c N(\log )}{16 \gamma^{3 / 2}\left(\varepsilon_{x, N} \varepsilon_{y, N}\right)^{3 / 4} \sigma_{s} \sigma_{p}^{3}}<\sigma_{H} f\left(\beta_{x}, \beta_{y}, \frac{\varepsilon_{x, N}}{\varepsilon_{y, N}}\right)> \\
& \frac{1}{\sigma_{H}^{2}}=\frac{1}{\sigma_{p}^{2}}+\left(\frac{H_{x}}{\varepsilon_{x, N}}+\frac{H_{y}}{\varepsilon_{y, N}}\right) \gamma \\
& H_{x, y}=\frac{1}{\beta_{x, y}}\left[\eta_{x, y}^{2}+\left(\beta_{x, y} \eta_{x, y}^{\prime}-\frac{1}{2} \beta_{x, y}^{\prime} \eta_{x, y}\right)^{2}\right]
\end{aligned}
$$

Notation: $\varepsilon_{x, N}, \varepsilon_{y, N}$ are the transverse normalized emittances, (log) is the Coulomb logarithm, $f$ is a function of its arguments and does not depend on the energy, <> represents an average over the lattice, $\eta_{x, y}$ and $\eta_{x, y}^{\prime}$ are the dispersion and its slope. The energy dependence of $\sigma_{H}$ is determined by the relative magnitudes of the first term and the two terms in parenthesis. At $450 \mathrm{GeV}$, these are of comparable magnitude. At higher energies the first term will determine the scaling of $\sigma_{H}$ since it scales as $\gamma^{3 / 2}$ while the other terms scale as $\gamma$. Hence we find that $\sigma_{H} \sim \gamma^{-3 / 4}$. Thus the energy scaling of the growth rate is $\frac{1}{T_{p}} \propto \gamma^{-3 / 2} \sigma_{s}^{-1} \sigma_{p}^{-3} \sigma_{H} \sim \gamma^{-3 / 2} \gamma^{1 / 4} \gamma^{9 / 4} \gamma^{-3 / 4} \sim \gamma^{1 / 4}$ 
The transverse growth rates are given by

$$
\frac{1}{T_{x, y}} \equiv \frac{1}{\sigma_{x, y}} \frac{d \sigma_{x, y}}{d t} \approx \frac{\sigma_{p}^{2}<H_{x, y}>\gamma}{\varepsilon_{x, y ; N}} \frac{1}{T_{p}} \sim \gamma^{-1 / 4}
$$

The longitudinal emittance growth rate will decrease as $\gamma^{1 / 2}$ while the horizontal emittances growth rate will fall as $\gamma^{-1 / 2}$. Hence increasing the injection energy from 450 $\mathrm{GeV}$ to $1.65 \mathrm{TeV}$ will increase the longitudinal emittance growth rate 1.9 times while the transverse emittance growth rate will fall by the same factor. The smaller transverse emittance at the start of the ramp may improve the ramp efficiency.

\section{REST-GAS SCATTERING}

The lifetime due to inelastic scattering with the residual gas will not be much affected at higher energy. The lifetime is determined by the cross-section of inelastic nuclear scattering and the gas density. This cross-section changes very little over the considered range of injection energies.

However the emittance growth rate due to elastic multiple scattering falls with increasing energy as $1 / \gamma$, so the emittance growth rate due to this effect will be abut a factor of 4 smaller at $1.65 \mathrm{TeV}$ compared to $450 \mathrm{GeV}$.

\section{CONCLUSIONS}

The most important factors in favor of increasing the injection energy are the increases in dynamic aperture, reduction in the persistent current decay and thereby the reduction in the setup time, the reduction in the energy spread and the reduction in the transverse emittance growth rate due to intra-beam and rest-gas scattering. The $\mathrm{TMCl}$ threshold intensity will also increase with increasing energy but the threshold intensity is about an order of magnitude above feasible bunch intensities. The microwave instability threshold decreases with energy but its threshold is also at least an order of magnitude above feasible intensities and not likely to be an issue. Effects due to space charge and synchrotron radiation will also likely play no role. Theoretical arguments suggest that electron cloud effects will also be less harmful at higher energy but the experimental situation is not so clear.

The negative impacts at higher injection energy are the smaller threshold for the loss of longitudinal Landau damping and the increase in the longitudinal emittance growth rate due to intra-beam scattering. At $1.65 \mathrm{TeV}$ with a somewhat pessimistic estimate for the longitudinal impedance, the threshold for Landau damping is $5.1 \times 10^{11}$ particles, comfortably above foreseen intensities for the upgrades. The higher longitudinal emittance growth rate at injection may not result in a longer bunch at top energy since the energy swing from injection to top energy is smaller which may reduce any blow up effects during the acceleration.

As mentioned in the introduction, two proposals for new injectors to the LHC have been presented. They differ in their maximum energy, likely reliability, ease of operation, cost and other details - a detailed comparison can be found in [1]. One way of deciding between the two is to ask whether the differences between injecting at $1 \mathrm{TeV}$ or at 1.65 
$\mathrm{TeV}$ are important. The momentum spread is more than $30 \%$ smaller at the higher energy and this itself is significant. Dynamic aperture will be higher and the setup time will be shorter at $1.65 \mathrm{TeV}$ but quantitative results need dedicated simulations and measurements. Experiments to settle the dependence of electron cloud issues on energy will also be very useful.

\section{REFERENCES}

[1] H. Piekarz, "Using Tevatron Magnets for HE-LHC or New Ring in LHC Tunnel", Proceedings of a Mini-Workshop on a High Energy LHC, Malta [2010]

[2] A.W. Chao and M. Tigner, "Handbook of Accelerator Physics and Engineering", World Scientific Press

[3] R. Assmann et al," Time dependent superconducting magnet errors and their effect on the beam dynamics at the LHC", Proceedings of EPACO2 (2002)

[4] F. Rugggiero, "Single beam collective effects in the LHC", Part Acc, 50, pg 83 (1995)

[5] W. Scandale, "LHC luminosity and energy upgrade", EPAC06, 2006

[6] J. Gareyte, "Accelerator Physics Issues at the LHC", EPAC96 (1996)

[7] D. Boussard et al.,"Is a longitudinal feedback system necessary for LHC?", LHC Project Report 205 (1999)

[8] LHC Design Report, CERN-2004-003

[9] G. Rumolo et al, "Dependence of the electron cloud instability on the beam energy", Phys. Rev. Lett., 100, 144801 (2008).

[10] K. Bane, "A simplified model of intra-beam scattering", Proceedings of EPACO2 (2002) 